\title{
SOME GEOMETRIC PROPERTIES OF WHITNEY STRATIFICATIONS
}

\author{
David Angelo Trotman ${ }^{\mathrm{a}^{*}}$ \\ ${ }^{a}$ Institut de Mathématiques de Marseille, Aix-Marseille Université, France \\ *Corresponding author: Email: david.trotman@univ-amu.fr \\ Article history \\ Received: June $30^{\text {th }}, 2021$ \\ Received in revised form: July $15^{\text {th }}, 2021 \mid$ Accepted: July $15^{\text {th }}, 2021$ \\ Available online: January $27^{\text {th }}, 2022$
}

\begin{abstract}
We describe some old and new results about Whitney stratifications, and state some open problems.

Keywords: Fibering; Stratifications; Triangulation; Whitney stratification.
\end{abstract}

DOI: http://dx.doi.org/10.37569/DalatUniversity.12.2.921(2022)

Article type: (peer-reviewed) Full-length research article

Copyright $@ 2022$ The author(s)

Licensing: This article is licensed under a CC BY-NC 4.0 
Let $V$ be an algebraic variety in $\mathbf{R}^{n}$ or $\mathbf{C}^{n}$. Then $\operatorname{Sing} V$, the singular or nonregular part of $V$ is an algebraic subvariety (Whitney, 1957). Thus, we derive the singular filtration by algebraic subvarieties

$$
V \supset \operatorname{Sing} V \supset \operatorname{Sing}(\operatorname{Sing} V) \supset \ldots
$$

The difference between two elements of the filtration is a smooth submanifold. We wish to impose conditions on the filtration so that these smooth pieces fit together well.

In 1965 Whitney (1965a, 1965b) proposed a certain condition (a) : suppose given two disjoint submanifolds $X$ and $Y$ such that $y_{0} \in Y \cap \bar{X}$. Then one says that the pair $(X, Y)$ satisfies condition (a), or that $(X, Y)$ is (a)-regular, if:

(a) given a sequence of points $x_{i}$ in $X$ that tends to $y_{0}$ as $i$ tends to infinity, such that the sequence of tangent spaces $T_{x_{i}} X$ tends to $\tau$ in the appropriate Grassmannian, then $T_{y_{0}} Y \subset \tau$.

This ensures a certain continuity of tangent spaces.

Example. Let $V=\left\{y^{2}=t^{2} x^{2}+x^{3}\right\} \subset \mathbf{R}^{3}$. Then $\operatorname{Sing} V=\{x=y=0\}$. The resulting filtration has the property that $(V-\operatorname{Sing} V$, Sing $V)$ satisfies condition (a). However, the local topology of $V$ at points of $\operatorname{Sing} V$ changes as one passes through $(0,0,0)$. Notice that tangent spaces to $V$ at points near $\operatorname{Sing} V$ different from $(0,0,0)$ nearly contain the secant $x \pi(x)$ where $\pi(x, y, t)=t$ is a projection. But near $(0,0,0)$ this is no longer true.

To avoid such a phenomenon, Whitney introduced a second condition (b) on a pair of disjoint submanifolds $(X, Y)$ at a point $y_{0} \in Y \cap \bar{X}$ :

(b) Given sequences $x_{i} \in X$ and $y_{i} \in Y$ both tending to $y_{0}$ as $i$ tends to infinity, and such that the tangent spaces $T_{x_{i}} X$ tend to $\tau$ and the secant lines $\overline{x_{i} y_{i}}$ tend to $\lambda$, then $\lambda \subset \tau$.

In particular, as a special case, $\lim \overline{x_{i} \pi\left(x_{i}\right)} \subset \tau$ : this is called condition $\left(b^{\pi}\right)$.

Exercise. (b) implies (a). Choose $y_{i}$ "far" from $\pi\left(x_{i}\right)$ such that $\overline{x_{i} y_{i}}$ tends to $\lambda \subset T_{y_{0}} Y$ for a given line $\lambda$ in $T_{y_{0}} Y$.

Using condition (b) we get the notion of a Whitney stratification.

Definition. A closed set $Z$ of a $C^{k}(1 \leq k \leq \infty$, or $k=\omega)$ manifold $M$ is said to have a $C^{k}$ Whitney stratification if there is a filtration by closed subsets

$$
Z=Z^{d} \supset Z^{d-1} \supset \cdots \supset Z^{1} \supset Z^{0} \supset \emptyset
$$

such that each $Z^{i}-Z^{i-1}$ is either empty or is a $C^{k}$ submanifold of dimension $i$, and the connected components of the $Z^{i}-Z^{i-1}$ (called the strata of dimension $i$ ) satisfy Whitney's condition (b). (If $X$ and $Y$ are strata with $y_{0} \in Y \cap \bar{X}$, then $(X, Y)$ satisfies condition (b) at $y_{0}$.)

For a detailed survey of the properties of Whitney stratifications and those satisfying other regularity conditions, see Trotman (2020). 
Proposition (Thom-Mather). If $X$ and $Y$ are distinct strata of a Whitney stratification and $Y \cap \bar{X} \neq \emptyset$, then $Y \subset \bar{X}-X$ (the frontier condition).

Theorem (Whitney). Every analytic variety admits a $C^{\omega}$ Whitney stratification.

This existence theorem remains true for semianalytic sets (Łojasiewicz, 1965), subanalytic sets (Denkowska et al., 1985; Hironaka, 1973; Łojasiewicz et al., 1986; Verdier, 1976), and also definable sets in o-minimal structures (Nguyen et al., 2014; Ta, 1998).

There are, however, simple examples of stratified sets occurring in dynamical systems that are not Whitney stratified; for example, the closure of the logarithmic spiral

$$
\left\{r=e^{-k t}, t \equiv \theta(\bmod 2 \pi)\right\}
$$

does not admit a Whitney stratification.

Although condition (a) is weaker than condition (b) it is often useful, as illustrated by the following characterization (Trotman, 1978/79).

Theorem (Trotman). Let $Z$ be a closed subset of a $C^{1}$ manifold $M$, with a stratification $\Sigma$. Then the following are equivalent :

(1) $\Sigma$ is (a)-regular (i.e. pairs of strata satisfy Whitney's condition (a)).

(2) For every $C^{1}$ manifold $N,\left\{f \in C^{1}(N, M) \mid f\right.$ is transverse to the strata of $\left.\Sigma\right\}$ is open in $C^{1}(N, M)$ with the Whitney $C^{1}$ topology.

See the book by Hirsch for the definition of the Whitney $C^{1}$ topology (Hirsch, 1976).

This theorem is useful for proving stability of classes of maps. The first proof that (1) implies (2) is due to Feldman (1965) while the proof that (2) implies (1) was in my $1977 \mathrm{PhD}$ thesis (Trotman, 1978/79). A new, simpler proof may be found in the 2013 $\mathrm{PhD}$ thesis of Trivedi (see Trivedi \& Trotman, 2014).

The most striking application of condition (b) is the Thom-Mather isotopy theorem (Mather, 2012; Thom, 1969).

Theorem (Thom-Mather). Every Whitney stratified set is locally topologically trivial along strata: if $(Z, \Sigma) \subset M$ is Whitney stratified and $x \in X$ where $X$ is a stratum, then there are an open neighborhood $U$ of $x$ in $M$ and a stratified homeomorphism

$$
h:(U, U \cap Z, U \cap X) \rightarrow(U \cap X) \times\left(\mathbf{R}^{k}, c L, \star\right)
$$

such that $p_{1} \circ h(z)=\pi(z) \in U \cap X$ where $p_{1}$ is projection to the first factor, $\pi$ is the pro- 
jection of a $C^{1}$ tubular neighborhood of $U \cap X, k=\operatorname{dim} M-\operatorname{dim} X$ and $L$ is a compact Whitney stratified set in the sphere $S^{k-1}$ called the link of $Z$ at $x$, with $c L$ the cone with base L and vertex $\star$.

One might hope to find a $C^{1}$ diffeomorphism. However this is not possible in general.

Example. Consider $V=\{x y(x-y)(x-t y)=0\} \subset \mathbf{R}^{3}$. The cross-ratio of four lines through the origin in a plane is a $C^{1}$ invariant and cannot change. Hence there does not exist a $C^{1}$ diffeomorphism transforming $V$ to a product even though $V$ is Whitney stratified along the $t$-axis.

However, there is a partial result due to Pawłucki (1985) that can sometimes be useful.

Theorem (Pawłucki). Let $X, Y$ be disjoint subanalytic submanifolds such that $\operatorname{dim} X=$ $\operatorname{dim} Y+1$. Then $(X, Y)$ is Whitney $(b)$-regular at every point $y$ of $Y$ if and only if there is a neighborhood $N$ of $y$ such that $N \cap(X \cup Y)$ is a finite union of $C^{1}$ manifolds-withboundary, and the common boundary is $N \cap Y$.

The equivalence remains true for definable sets in polynomially bounded o-minimal structures (Trotman \& Valette, 2017). However, in the non-polynomially bounded case it fails as shown by the next example.

Example. Let $Z=\overline{\left\{y=z^{x^{2}+1}, z>0\right\}} \subset \mathbf{R}^{3}$. Let $Y$ be the $x$-axis and $X=Z-Y$. Then the pair of strata $(X, Y)$ is (b)-regular, but does not form a $C^{1}$ manifold-with-boundary. Adding the half-plane $\{y=0\}$ and filling in, we also obtain a 3-dimensional example of a (b)-regular definable stratified set for which the density is not continuous along a stratum whereas the density is always continuous along strata of a (b)-regular definable stratified set if the o-minimal structure is polynomially bounded (Nguyen \& Valette, 2016).

What lies between homeomorphisms and $C^{1}$ diffeomorphisms?

One answer is bi-lipschitz homeomorphisms (Parusiński, 1994). Here we have an analogue of the Thom-Mather isotopy theorem.

Theorem (Parusiński). Subanalytic sets admit (Whitney) stratifications which are locally bilipschitz trivial along strata.

This result is also true for definable sets in polynomially bounded o-minimal structures (Halupczok \& Yin, 2018; Nguyen \& Valette, 2016).

These stratifications have a rather strong regularity property due to T. Mostowski. He called them Lipschitz stratifications. They are characterized by the possibility of ex- 
tending Lipschitz vector fields on (the closure of a stratum) to neighbouring strata, with control of the Lipschitz constants.

In the non-polynomially bounded case, Parusinski's theorem of generic bilipschitz triviality fails: the previous example shows this. Or, more simply, consider $Z=\{y=$ $0\} \cup \overline{\left\{y=z^{x}, z>0\right\}} \subset \mathbf{R}^{3}$. The Lipschitz type varies continuously.

Even for semialgebraic sets, (b)-regularity does not imply bi-lipschitz triviality.

Example (Koike). Let $Z=\overline{\left\{y^{2}=t^{2} x^{2}+x^{3}, x>0\right\}} \subset \mathbf{R}^{3}$. Setting $Y$ to be the $t$-axis, and $X=Z-Y$, one finds easily that $(X, Y)$ is Whitney $(b)$-regular, but the bilipschitz type of the slice $\{t=$ constant $\}$ with $Z$ at $t=0$ is distinct from the bilipschitz type for $t \neq 0$.

Question. How strong can one make the conclusion to the Thom-Mather isotopy theorem?

Using the conclusion to the Thom-Mather isotopy theorem, let us write

$$
h(z)=(\pi(z),[\rho(z), \theta(z)]) \in(U \cap X) \times\left(\mathbf{R}^{k}, c L, \star\right),
$$

where $\pi: T_{X} \rightarrow X$ is the projection/retraction of a tubular neighborhood $T_{X}$ and $\rho: T_{X} \rightarrow$ $[0, \varepsilon)$ is a tubular function (or distance to $X$ ) such that $\rho^{-1}(0)=X$.

The proof of the isotopy theorem involves integrating stratified controlled lifts of vector fields on $X$. A controlled lift of a vector field $v_{X}$ on $X$ is the assignment of a vector field $v_{Y}$ on each stratum $Y$ of $\Sigma$ in a tubular neighbourhood $T_{X}$ of $X$ such that $\pi_{*} v_{Y}=v_{X}$ and $\rho_{*} v_{Y}=0$.

Saying $\pi_{*} v_{Y}=v_{X}$ means that the differential of $\pi$ maps $v_{Y}$ to $v_{X}$.

Saying $\rho_{*} v_{Y}=0$ means that $v_{Y}$ is tangent to the level hypersurfaces of $\rho$.

Saying that $v=\left\{v_{Y}\right\}$ is stratified means that each $v_{Y}=\left.v\right|_{Y}$ is tangent to $Y$.

Question. Can the lifts $\left\{v_{Y}\right\}_{Y \in \Sigma}$ be made continuous as we change strata? This question was posed already in 1969 by Thom (1969) and later by Mather (2012).

Answer. Yes (Shiota, 1996; and independently, du Plessis, 1999).

Knowing that the controlled vector fields can be chosen to be continuous suggests asking for continuity of the tangent spaces to the leaves $\{z \mid \theta(z)=\theta, \rho(z)=\rho\}$ for fixed $\theta$ and $\rho$ (each of which is diffeomorphic to $U \cap X$ ), as we change strata. This was proved by Murolo, du Plessis and myself in 2017 (Murolo et al., 2017), resolving the smooth Whitney fibering conjecture, and giving a new proof of the Thom-Mather isotopy theorem. The proof works in fact for the weaker regularity condition called $(c)$-regularity due 
to Karim Bekka and thus also provides a new proof of Bekka's theorem (1991) stating that $(c)$-regular stratified sets are locally topologically trivial.

Theorem (Murolo-du Plessis-Trotman). In the proof of the Thom-Mather isotopy theorem, $h$ can be chosen to ensure continuity of tangent spaces to the leaves $\{z \mid \theta(z)=$ $\theta, \rho(z)=\rho\}$.

Whitney's 1965 holomorphic fibering conjecture (Whitney, 1965a) asks for a stratification of a given analytic variety such that the leaves defined by a trivializing homeomorphism be holomorphic submanifolds with their tangent spaces varying continuously as one approaches a given stratum. This was proven in 2017 with many extra conditions (including $h$ subanalytic and arc-analytic) by Parusiński and Pãunescu (2017) in the local analytic and global algebraic, real and complex cases.

Open problem. Prove the semi-algebraic (or subanalytic or definable) version of the Whitney fibering conjecture.

\section{Triangulations.}

Conjecture (Thom). Every Whitney stratified set admits a Whitney triangulation (i.e., the open simplexes are the strata of a Whitney stratification refining the given one).

One reason for the interest in this conjecture is that it would imply that the homology of a Whitney stratified set is supported by Whitney stratified cycles (a conjecture of Goresky (1981)).

Shiota (2005) has proved that every semialgebraic set admits a semialgebraic Whitney triangulation, while the case of definable sets in o-minimal structures was proved by Czapla (2012) in her thesis at the Jagellonian University in Cracow.

Weak Conjecture. Every Whitney stratified set admits a Whitney cellulation, i.e. the open cells are the strata of a Whitney stratification.

This would also imply Goresky's conjecture.

\section{REFERENCES}

Bekka, K. (1991). C-régularité et trivialité topologique [C-regularity and topological triviality]. In D. Mond \& J. Montaldi (Eds.), Singularity theory and its applications, Warwick 1989, Part I: Geometric aspects of singularities (Lecture Notes in Mathematics) (pp. 42-62). Springer. https://doi.org/10.1007/BFb0086373

Czapla, M. (2012). Definable triangulations with regularity conditions. Geometry \& Topology, 16(4), 2067-2095. https://doi.org/10.2140/gt.2012.16.2067

Denkowska, Z., Wachta, K., \& Stasica, J. (1985). Stratification des ensembles sous-analy- 
tiques avec les propriétés (A) et (B) de Whitney [Stratification of sub-analytical sets with Whitney properties (A) and (B)]. Universitatis Iagellonicae Acta Mathematica, 25, 183-188.

du Plessis, A. A. (1999). Continuous controlled vector fields. In W. Bruce \& D. Mond, Singularity theory (Liverpool, 1996), London Mathematical Society Lecture Note Series, 263 (pp. 189-197). Cambridge University Press. https://doi.org/10.1017/C BO9780511569265.013

Feldman, E. A. (1965). The geometry of immersions. I. Transactions of the American Ma -thematical Society, 120, 185-224. https://doi.org/10.1090/S0002-9947-1965-01 85602-6

Goresky, R. M. (1981). Whitney stratified chains and cochains. Transactions of the American Mathematical Society, 267, 175-196. https://doi.org/10.1090/S0002-9947-1 981-0621981-X

Halupczok, I., \& Yin, Y. (2018). Lipschitz stratifications in power-bounded o-minimal fields. Journal of the European Mathematical Society, 20(11), 2717-2767. https: //doi.org/10.4171/JEMS/823

Hironaka, H. (1973). Subanalytic sets. In Y. Kusunoki (Ed.), Number theory, algebraic geometry and commutative algebra, in honor of Yasuo Akizuki (pp. 453-493). Kinokuniya.

Hirsch, M. W. (1976). Differential Topology. Springer. https://doi.org/10.1007/978-1-4 684-9449-5

Łojasiewicz, S. (1965). Ensembles semi-analytiques [Semi-analytical sets]. Institut des Haute Études Scientiques Notes.

Łojasiewicz, S., Stasica, J., \& Wachta, K. (1986). Stratifications sous-analytiques. Condition de Verdier [Sub-analytic stratifications. Verdier condition]. Bulletin Polish Academy of Sciences Mathematics, 34(9-10), 531-539.

Mather, J. N. (2012). Notes on topological stability. Bulletin of the American Mathematical Society (New Series), 49(4), 475-506. https://doi.org/10.1090/S0273-09792012-01383-6

Murolo, C., du Plessis, A., \& Trotman, D. (2017). On the smooth Whitney fibering conjecture, preprint. https://hal.archives-ouvertes.fr/hal-01571382

Nguyen, N., \& Valette, G. (2016). Lipschitz stratifications in o-minimal structures. Annales Scientifique de L'École Normale Superieure, 49(4), 399-421. https://doi.org/10 .24033/asens.2286

Nguyen, N., \& Valette, G. (2018). Whitney stratifications and the continuity of local Lipschitz-Killing curvatures. Annales de l'Institut Fourier (Grenoble), 68(5), 22532276. https://doi.org/10.5802/aif.3208

Nguyen, N., Trivedi, S., \& Trotman, D. (2014). A geometric proof of the existence of definable Whitney stratifications. Illinois Journal of Mathematics, 58(2), 381-389. https://doi.org/10.1215/ijm/1436275489

Parusiński, A. (1994). Lipschitz stratification of subanalytic sets. Annales Scientifiques de l'École Normale Superieure, 27(6), 661-696. https://doi.org/10.24033/asens. 
1703

Parusiński, A., \& Pãunescu, L. (2017). Arc-wise analytic stratification, Whitney fibering conjecture and Zariski equisingularity. Advances in Mathematics, 309, 254-305. https://doi.org/10.1016/j.aim.2017.01.016

Pawłucki, W. (1985). Quasiregular boundary and Stokes's formula for a subanalytic leaf. In J. Ławrynowicz (Ed), Seminar on deformations (Lódz/Warsaw, 1982/84) Lecture Notes in Mathematics, 1165, (pp. 235-252). Springer. https://doi.org/10.100 7/BFb0076157

Shiota, M. (1996). Geometry of subanalytic and semialgebraic sets. Birkhäuser. https://doi .org/10.1007/978-1-4612-2008-4

Shiota, M. (2005). Whitney triangulations of semialgebraic sets. Annales Polonici Mathematici, 87, 237-246. https://doi.org/10.4064/ap87-0-20

Ta, L. L. (1998). Verdier and strict Thom stratifications in o-minimal structures. Illinois Journal of Mathematics, 42(2), 347-356. https://doi.org/10.1215/ijm/1256045049

Thom, R. (1969). Ensembles et morphismes stratifiés [Sets and stratified morphisms]. $B u$ -lletin of the American Mathematical Society, 75, 240-284. https://doi.org/10.1090 /S0002-9904-1969-12138-5

Trivedi, S., \& Trotman, D. (2014). Detecting Thom faults in stratified mappings. Kodai Mathematical Journal, 37(2), 341-354. https://doi.org/10.2996/kmj/1404393891

Trotman, D. (1978/79). Stability of transversality to a stratification implies Whitney (a)regularity. Inventiones Mathematicae, 50(3), 273-277. https://doi.org/10.1007/BF 01410081

Trotman, D. (2020). Stratification theory. In J. L. Cisneros-Molina, T. D. Lê, \& J. Seade (Eds.), Handbook of geometry and topology of singularities I (pp. 231-260). Springer. https://doi.org/10.1007/978-3-030-53061-7_4

Trotman, D., \& Valette, G. (2017). On the local geometry of definably stratified sets. In F. Broglia, F. Delon, M. Dickman, D. Gondard-Cozette, \& V. A. Powers, (Eds.), Ordered algebraic structures and related topics. Contemporary Mathematics, 697 (pp. 349-366). American Mathematical Society. https://doi.org/10.1090/con $\mathrm{m} / 697 / 14061$

Verdier, J.-L. (1976). Stratifications de Whitney et théorème de Bertini-Sard [Whitney stratifications and the Bertini-Sard theorem]. Inventiones Mathematicae, 36, 295312. https://doi.org/10.1007/BF01390015

Whitney, H. (1957). Elementary structure of real algebraic varieties. Annals of Mathematics, 66(3), 545-556. https://doi.org/10.2307/1969908

Whitney, H. (1965a). Local properties of analytic varieties. In S. S. Cairns (Ed.), Differential and Combinatorial Topology: A Symposium in Honor of Marston Morse (pp. 205-244). Princeton University Press. https://doi.org/10.1515/97814008748 42-014

Whitney, H. (1965b). Tangents to an analytic variety. Annals of Mathematics, 81(3), 496-549. https://doi.org/10.2307/1970400 